\title{
Measurement of the cosmic muon charge asymmetry
}

\author{
Jónatan Piedra*† \\ University of Florida \\ E-mail: piedra@cern.ch
}

In late 2008, CMS collected 300 million events from atmospheric cosmic ray muons in a dedicated long run, with the full detector operational assembled in its definitive place along the LHC ring. Using these data, CMS aims to measure the charge asymmetry of cosmic muons, as a function of the muon momentum, from approximately $100 \mathrm{GeV} / \mathrm{c}$ up to the TeV/c scale. While the analysis of cosmic muons is not part of the physics program of CMS, it requires high quality measurements that demand good performance of the CMS detector, from the data taking to the reconstruction and analysis software.

European Physical Society Europhysics Conference on High Energy Physics, EPS-HEP 2009,

July 16 - 222009

Krakow, Poland

\footnotetext{
* Speaker.

${ }^{\dagger}$ On behalf of the CMS Collaboration.
} 

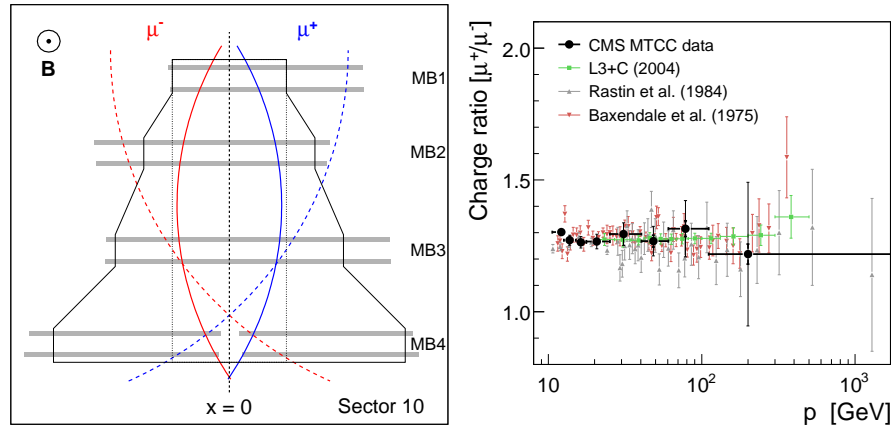

Figure 1: (Left) Definition of the left-right symmetric fiducial geometry in the muon system. (Right) Muon charge ratio measured by CMS (black dots) with statistical (thick bars) and systematic (thin bars) errors, together with results from other experiments.

\section{Introduction}

Many particles like the positron, muon, pion and kaon were discovered by observing cosmic rays. It is in the interaction of primary cosmic rays (protons, neutrons) with atmospheric nuclei where pions, kaons and then muons are produced, via $\pi^{ \pm}, K^{ \pm} \rightarrow \mu^{ \pm}+v_{\mu}\left(\overline{v_{\mu}}\right)$. The goal of this analysis is to measure the cosmic muon charge ratio between $\mu^{+}$and $\mu^{-}$as a function of the muon momentum at the Earth's surface. Other dedicated experiments have already measured this ratio [1]. We aim at adding information in the 400-1000 GeV momentum range, where few data points are yet available.

\section{Magnet Test and Cosmic Challenge Data}

The Magnet Test and Cosmic Challenge (MTCC) [2] took place in the second half of 2006. Its main goal was to test operation of the CMS magnet before it was lowered to the experimental cavern. It also allowed testing the CMS detector using cosmic-ray muons. In this period 15 million events were triggered by the muon system, with the magnetic field at $4 \mathrm{~T}$. The enabled read-out consisted of sectors 10 and 11, which accounted for a $60^{\circ}$ slice of CMS in the $r \phi$ plane. In the $z$ direction 2 out of 5 wheels were part of the read-out, together with adjacent end-cap disks. These data had provided the first measurement of a physical quantity performed by the CMS experiment []].

Due to the magnetic field, unlike-sign charged particles bend in opposite directions in the $r \phi$ plane, populating different regions of the detector and thus introducing a bias in the relative detector efficiency of $\mu^{+}$and $\mu^{-}$. In order to avoid such bias, the fiducial geometric acceptance of the detector was constrained to be left-right symmetric, as shown in Figure 1. Then a high-quality sample of muons was selected as described in [3] yielding the result shown in Figure 1, which nicely compared to more precise experiments.

\section{Cosmic Run At Four Tesla Data}

With the CMS [ $₫$ ] detector already $100 \mathrm{~m}$ underground and fully operational, in the months of October and November 2008, about 370 million cosmic muon events were collected in the Cosmic 
Run At Four Tesla (CRAFT). It was a 24/7 data-taking period for detector commissioning, with the magnetic field at $3.8 \mathrm{~T}$. The rest of this paper is dedicated to momentum resolution and charge studies performed on this data sample.

\section{Analysis Components}

The CMS detector is not designed as a cosmic ray detector, with the typical geometry of a collider experiment, and shielded as it is from cosmic rays by being almost $100 \mathrm{~m}$ underground. Besides, the amount of material shielding CMS changes across the detector volume. As a collider experiment it was designed to expect particles from the beam spot, which means that the trigger and read-out timing of the detector had to be modified for cosmic muons data-taking. In addition, the tracker system, which provides the best momentum resolution measurements, is a small target for the incident cosmic rays to hit.

The process to measure the charge ratio between $\mu^{+}$and $\mu^{-}$as a function of the muon momentum at the Earth's surface starts inside the detector, where the charge ratio as a function of the reconstructed transverse momentum is measured. The difficulty of this analysis, including the propagation from the center of CMS to the Earth's surface, is related to the identification of the possible biases that could affect the measurement, and eventually correct for them. The reconstruction of cosmic muons is performed using specialized algorithms [5], as the standard collisions reconstruction is not optimal for this analysis (assumes muons emanating from the interaction point.) Among the specialized reconstruction algorithms two have been chosen to perform the charge ratio analysis. The following sections describe some features of these algorithms and include preliminary performance results.

\subsection{1-leg Stand-Alone Muon Reconstruction}

This Stand-Alone (SA) muon algorithm reconstructs muons considering only the information from the muon system, providing a higher statistics sample than that used in the second approach, which requires tracker information and the smaller tracker volume limits the sample size. In addition, a cosmic muon entering the CMS detector is reconstructed as a single track in the whole detector (upper and lower hemispheres included.) The use of a single object provides a longer lever arm and therefore good resolution is expected at high transverse momentum. The transverse momentum resolution is estimated by comparing the measurements given by two independent systems - the muon chambers and the tracker. In particular, we compare the curvature or inverse of the transverse momentum. The curvature difference (normalized by the curvature in the tracker) is fitted with a Gaussian distribution in bins of transverse momentum, and the results for the mean and the width of the fits can be seen in Figure 2, for both data and Monte Carlo simulation. The mean results for data and Monte Carlo are both within 1\%, which corresponds to an excellent agreement between the muon system and the inner tracker. Another key issue is the determination of the mismeasurement of the muon charge. To estimate how often the charge is correctly determined, the results given by the muon system alone and the tracker are compared. The fraction of times that both systems disagree in the assignment of the muon charge is shown in Figure 2, where the agreement is excellent (below 5\%) up to $p_{T}=500 \mathrm{GeV}$. 

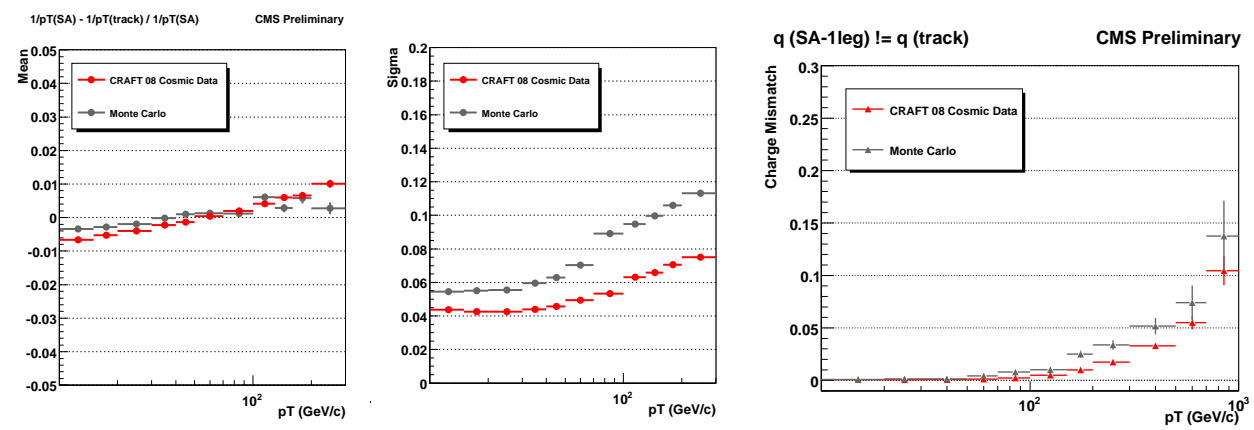

Figure 2: 1-leg reconstruction performance plots. Data points are in red and MC points are in grey. (Left and center) Transverse momentum resolution. (Left) mean of the Gaussian fits. (Center) Width of the Gaussian fits. (Right) Charge mismatch.

\subsection{2-leg Reconstruction}

This algorithm reconstructs muons including the tracker information, therefore the sample size will be limited by the tracker volume. It splits the entering cosmic muon at the point of closest approach to the center of CMS. In other words, a cosmic muon is naturally divided into an upper and a lower leg. This allows a completely data-driven approach, where the charge ratio is measured as a function of the average curvature $C$ and the resolution is measured from the curvature difference $d_{C}$ as defined in Equation 4.1 .

$$
C \equiv \frac{1}{2}\left(\frac{q^{u p}}{p_{T}^{u p}}+\frac{q^{l o w}}{p_{T}^{l o w}}\right), \quad d_{C} \equiv \frac{1}{2}\left(\frac{q^{u p}}{p_{T}^{u p}}-\frac{q^{l o w}}{p_{T}^{l o w}}\right)
$$

To estimate the resolution, $d_{C} / C$ is fitted with Gaussian distributions in average curvature bins. The width of the fits in Figure 3 shows an excellent resolution (below 5\%) in all the curvature spectrum. To check how well this resolution estimator reproduces the reality we compare in the Monte Carlo simulation the resolution given by $d_{C}$ and the true resolution, defined as the difference between the reconstructed and the simulated curvatures. Figure 3 shows an excellent agreement between both variables. The fraction of times that the upper and the lower leg disagree in the assignment of the muon charge is shown in Figure 3. This fraction stays below 3\% up to $p_{T}=$ $300 \mathrm{GeV}$.

\section{Systematics}

We have found that the charge ratio measurement is affected by the following systematic uncertainties: muon selection, magnetic field map, detector alignment and trigger efficiency. Each of them has been estimated for the 2-leg reconstruction algorithm, and the results are briefly described.

The muon selection is aimed at gathering a sample of high quality muons without introducing any bias. This systematic uncertainty (below 1\%) was estimated by checking the result of removing each cut. As for the systematic effect due to the magnetic field, we have compared the impact of different field maps in the analysis, getting the worse disagreement (3\%) at high $p_{T}$. Same approach has been followed to estimate the effect of the muon, tracker and muon-tracker relative alignments 

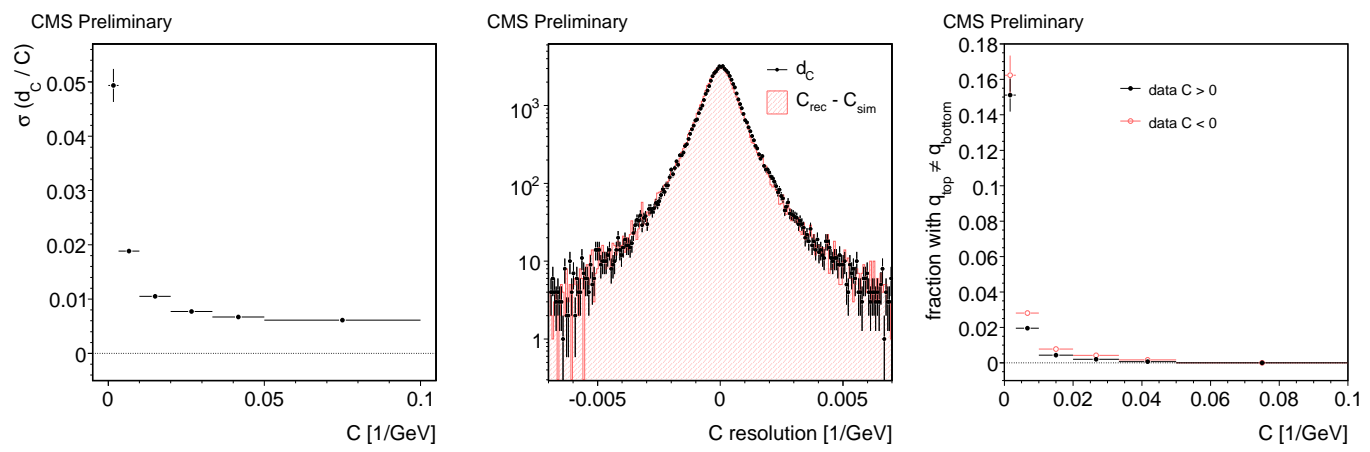

Figure 3: 2-leg reconstruction performance plots. (Left) Width of the Gaussian fits of the curvature resolution. (Center) Comparison of the resolution estimator $d_{C}$ (black points) with the true resolution (dashed red histogram). (Right) Charge mismatch.

in the curvature values, finding the disagreement between different alignments to be up to 5\% at high $p_{T}$. Finally, we checked if the trigger efficiency was different for positive and negative muons, obtaining an excellent agreement for positive and negative muons, both for data and Monte Carlo simulation.

\section{Conclusions}

With the cosmic data accumulated by CMS in the CRAFT commissioning run during the Autumn of 2008 several improvements have been achieved, especially in the understanding of the CMS magnetic field map and the alignment of the different systems. With this data two charge ratio analyses have gathered high quality muons after an unbiased selection. In particular, the 2leg analysis is fully data-driven, with the resolution proxy checked on Monte Carlo simulation. Besides, several systematic uncertainties have been already studied, finding no strong bias for any of them.

\section{References}

[1] PRD 76, 052003 (2007), P. Adamson et al., "Measurement of the atmospheric muon charge ratio at TeV energies with the MINOS detector"

[2] CMS Note 2007/005, CMS Collaboration, "The CMS Magnet Test and Cosmic Challenge (MTCC Phase I and II) Operational Experience and Lessons Learnt"

[3] CMS Note 2008/016, M. Aldaya and P. Garcia-Abia, "Measurement of the charge ratio of cosmic muons using CMS data"

[4] JINST 3:S08004 (2008), CMS Collaboration, "The CMS experiment at the CERN LHC"

[5] Eur. Phys. J. C 56, 449-460 (2008), C. Liu et al., "Reconstruction of cosmic and beam-halo muons with the CMS detector" 